\title{
Uncertainty about spatial frequency, spatial position, or contrast of visual patterns
}

\author{
ELIZABETH T. DAVIS, PATRICIA KRAMER, and NORMA GRAHAM \\ Columbia University, New York, New York
}

\begin{abstract}
Prevalent theories of pattern vision postulate mechanisms selectively sensitive to spatial frequency and position but not to contrast. Decreased performance in the detection of visual stimuli was found when the observer was uncertain about the spatial frequency or spatial position of a patch of sinusoidal grating but not when he was uncertain about contrast. The uncertainty effects were consistent with multiple-band models in which the observer is able to monitor perfectly all relevant mechanisms. Performance deteriorates when the observer must monitor more mechanisms, because these mechanisms are noisy and give rise to false alarms. This consistency is further evidence that the spatial-frequency and spatial-position mechanisms are noisy, a conclusion previously suggested by the "probability summation" demonstrated in the thresholds for compound stimuli. Somewhat paradoxically, the Quick pooling model, which quantitatively accounts for the amount of probability summation in pattern thresholds, predicts no effects of uncertainty. It cannot, therefore, be strictly correct.
\end{abstract}

Current theories of pattern vision assume the existence of mechanisms (often called channels) selectively sensitive to different spatial frequencies and also of mechanisms selectively sensitive to different spatial positions. A possible physiological substrate for a spatial-frequency channel is an array of neurons having receptive fields all of the same size and orientation but located at different positions within the visual field. A possible physiological substrate for a mechanism sensitive to a particular spatial position is the set of receptive fields located at that position. (See Graham, 1981, for a review.)

A particular quantitative version of this theory has been extremely successful in predicting the thresholds for a wide variety of patterns (e.g., Bergen, Wilson, \& Cowan, 1979; Graham, 1977; Graham, Robson, \& Nachmias, 1978; Mostafavi \& Sakrison, 1976; Quick, Mullins, \& Reichert, 1978; Robson \& Graham, 1981; Watson, 1982; Wilson \& Bergen, 1979). Although a name for this model has not become standard, we will call it the Quick pooling model since its current use in vision originates with Quick (1974) and it is much quicker to use than alternative models.

The Quick pooling model, as derived from assumptions of independent variability in the responses of each of the multiple mechanisms, is the pure form of a high-threshold model in which the possibility of a false alarm in any mechanism is actually zero.

This research was partially supported by NSF Grant BNS-7618839 to Norma Graham. We wish to thank Terry Schile for being such a helpful observer and research assistant. We are grateful to Jacob Nachmias and Bill Friedman for useful comments on an earlier draft of this manuscript. Requests for reprints should be sent to Norma Graham, Department of Psychology, Columbia University, New York, New York 10027.
High-threshold models (e.g., Green \& Weber, 1980) predict no effect of uncertainty. Uncertainty effects, however, have been reported for the spatial frequency of sinusoidal gratings (e.g., Davis \& Graham, 1981; Graham, Robson, \& Nachmias, 1978) and for spatial position of aperiodic visual targets (e.g., Cohn \& Lasley, 1974; Pelli, 1981; Posner, 1978; Posner, Snyder, \& Davidson, 1980).

The failure of the Quick pooling model to predict uncertainty effects seemed unimportant when the interpretations of experimental results depended on differences that were substantially larger than the uncertainty effects. Now, however, as the field has become more refined, the interpretation of experimental results is affected by uncertainty effects. (See, for example, the difference between the results in Graham \& Nachmias, 1971, and those in Graham, Robson, \& Nachmias, 1978.) Gaining a better understanding of these uncertainty effects, therefore, is critical for further development of these models of pattern vision. This study addresses several issues in the hope of contributing to such an understanding.

(1) Although the Quick pooling model does not predict uncertainty effects, other models assuming multiple mechanisms do (Ball \& Sekuler, 1980; Creelman, 1960; Green \& Swets, 1966; Green \& Weber, 1980). These models are commonly called single-band and multiple-band models and are described further in the results section.

To determine whether single-band or multipleband models (or perhaps neither) could explain the effects of uncertainty about spatial frequency, the detectability of several widely separated spatial frequencies was measured under conditions of both uncertainty and certainty using a temporal two-alternative 
forced-choice procedure. In these experiments, the separation between the closest frequencies was a factor of either two or four, far enough apart to stimulate completely separate channels (e.g., Watson, 1982). Earlier studies (Davis, 1981; Davis \& Graham, 1981) have used closely spaced frequencies in order to investigate the spatial frequency tuning of the channels. Predictions like those tested in this study for the magnitudes of uncertainty effects could not be done in those earlier studies without knowing the precise bandwidths and degree of additivity of the spatial frequency channels. Furthermore, the overall conditions of the earlier experiments were unlike those in the summation experiments in which the Quick pooling model has been so successful."

(2) Evidence from pattern thresholds also suggests that the mechanisms sensitive to different spatial positions are independently noisy in precisely the same way as are mechanisms sensitive to different spatial frequencies (e.g., Robson \& Graham, 1981). According to multiple-band models, such noise should lead to uncertainty effects of spatial position. In any case, simple stimuli such as patches of sinusoidal grating at different positions are commonly used stimuli in present investigations of pattern vision. Uncertainty effects for position are, therefore, of practical consequence in interpreting the results of these experiments. Previous studies using aperiodic stimuli have disagreed on the magnitude (and even on the existence) of effects of spatial-position uncertainty, perhaps due to the different processing induced by different stimuli and task demands.

We, therefore, measured the detectability of nonoverlapping patches of grating in conditions of both uncertainty and certainty using a forced-choice procedure. As it turned out, uncertainty about spatial position did decrease detectability. So, for these results also, we asked the question of whether singleband or multiple-band (or perhaps neither) could account for the obtained uncertainty effect.

(3) Current theories of pattern vision do not ordinarily assume mechanisms selectively sensitive to different contrast (intensity) values, although mechanisms selective for different intensities of auditory tones have been suggested (Green \& Luce, 1974; Luce, Green, \& Weber, 1976). Therefore, one might not expect to find an uncertainty effect for contrast. It is of practical importance, however, to know whether intermixing gratings of different contrasts (but otherwise identical) leads to decreased detectability. We therefore measured the detectability of gratings of different contrast under conditions of both uncertainty and certainty, using a forced-choice procedure.

(4) In all the experiments reported here, there were not only intermixed blocks (containing trials of several different stimuli-the uncertainty condition) and alone blocks (containing trials of only one stimulus - the certainty condition), but also a third kind of block-cued intermixed. In these cued intermixed blocks, an auditory cue occurred at the beginning of each trial (approximately $750 \mathrm{msec}$ before the first of the two forced-choice intervals) indicating which stimulus would be presented on that trial. It is of some interest to know whether such a cue would eliminate the uncertainty effect of whether it takes longer than $750 \mathrm{msec}$ for an observer's behavior to be modified. In previous studies, precues have been effective in reducing or eliminating the effect of uncertainty about auditory frequency (Gilliom \& Mills, 1976; Swets \& Sewall, 1961) as well as about direction of motion (Ball \& Sekuler, 1981).

Furthermore, if the cues worked to eliminate the uncertainty effects in this study (as, indeed, they did), the comparison between cued and uncued intermixed blocks would bypass a problem inherent in the comparison between alone and uncued intermixed blocks-the problem of possible differential adaptation to patterns. (Averaged over time, the visual stimulation in an alone block is quite different from that in an uncued intermixed block.) Differential pattern adaptation certainly does occur in some circumstances. After observers have adapted to gratings, they are less sensitive to gratings of the same frequency (and position) (e.g., Blakemore \& Campbell, 1969; Pantle \& Sekuler, 1968). The amount of desensitization increases with the contrast of the adapting gratings, but some desensitization is found even with adapting gratings of very low contrast. If differential adaptation occurred here, it would tend to decrease performance in alone blocks relative to intermixed blocks, thereby diminishing the size of the uncertainty effect measured by comparing alone blocks with (uncued) intermixed ones. However, the visual stimulation in both the cued and uncued intermixed blocks is identical, on the average.

\section{METHOD}

In all the experiments reported here, the detectability of each stimulus was measured by a temporal two-interval forced-choice procedure in three different types of blocks. In the alone blocks, the same stimulus appeared on every trial throughout a subblock so the observer was certain as to which stimulus would appear. In the uncued intermixed blocks, trials of several different stimuli were intermixed randomly within a block so that the observer could not know which stimulus would appear on each trial. In the cued intermixed blocks, trials of several stimuli were again randomly intermixed within a block, but each trial contained an auditory cue that came about .75 sec before the two forced-choice intervals and informed the observer which particular stimulus would appear on that trial.

\section{Stimall}

The stimuli were all vertically oriented gratings presented on the face of an oscilloscope with an annular surround of the same mean luminance (1.9 fL). Each stimulus was presented for $100 \mathrm{msec}$ with an abrupt onset and offset. At least five cycles were presented in 
each sinusoidal grating. Short, vertical lines on the annular surround above and below the center of the oscilloscope face served as fixation marks. Other details of the stimulus display are described in Davis and Graham (1981).

In each experiment, the stimuli varied along one of the three dimensions: spatial frequency, spatial position, or contrast.

Spatial frequency. In the spatial-frequency uncertainty experiments, either three or five spatial frequencies were used. The spatial frequencies were always at least one octave apart so that each grating would maximally stimulate a different set of spatial frequency channels. (See Figure 1 for exact values.) In these experiments each grating filled the entire display, which was 10.5 deg horizontally $\times 8$ deg vertically at a viewing distance of $72.5 \mathrm{~cm}$ for Observer P.K. and $5.25 \times 4 \mathrm{deg}$ at a distance of $145 \mathrm{~cm}$ for T.S. On the basis of previous testing, the contrasts at each spatial frequency were chosen to produce approximately equal performance in alone blocks. (For Observer P.K., the nominal contrasts at 2 and 4 cycles/deg (cpd) were .30 and $.50 \mathrm{log}$ units higher, respectively, than the contrast at $1 \mathrm{cpd}$. For Observer T.S. in the three-frequency experiment, the contrasts at 4 and $16 \mathrm{cpd}$ were .05 and $1.20 \mathrm{log}$ units higher, respectively, than at $1 \mathrm{cpd}$, and in the five-frequency experiment, the contrasts at $2,4,8$, and $16 \mathrm{cpd}$ were .05, .10, .55, and $1.25 \log$ units higher, respectively, than the contrast at $1 \mathrm{cpd}$.) These nominal contrasts were computed from the amplitude of the signal going into the oscilloscope. The oscilloscope will attenuate high frequencies somewhat, however, so the true contrasts at high frequencies will be slightly lower than the nominal contrasts.

Spatial position. In the spatial-position uncertainty experiments, there were three adjacent, but nonoverlapping, positions (left, center, or right). Each stimulus was a seven-cycle patch of sinusoidal grating. If the three patches had been presented simultaneously, they would have formed a continuous grating which filled the face of the display. The viewing distance was $72.5 \mathrm{~cm}$ for Observer P.K. and $145 \mathrm{~cm}$ for Observer T.S., producing stimulus spatial frequencies of 2 and $4 \mathrm{cpd}$, respectively. The contrasts at each position were chosen to produce approximately equal performance in the alone blocks. (The contrasts of the side patches were .05 log units higher than the contrast of the center for $\mathrm{Ob}$ server P.K. and .10 log units higher for T.S.)

Contrast. In the contrast uncertainty experiments, three contrasts were used. The middle contrast was chosen to yield approximately $75 \%$ correct detection in the alone blocks. The contrasts were spaced .2 or $.3 \mathrm{log}$ units apart. The 4-cpd gratings used in these experiments filled the entire $5.25 \times 4$ deg display and were viewed from a distance of $145 \mathrm{~cm}$ for both observers, T.S. and E.D.

\section{Procedures}

Within each experiment, there were three types of blocks: alone, uncued intermixed, and cued intermixed.

An alone block was composed of several subblocks, one for each stimulus. Throughout a subblock, the same stimulus was presented on every trial and the order of subblocks within a block was chosen randomly. Usually there were three subblocks of 100 trials each, except for the five-frequency experiment, in which there were five subblocks of 60 trials each.

An uncued intermixed block was composed of trials of the several stimuli randomly intermixed within a block. Each block contained 300 trials, with 100 repetitions of each of three stimuli, except for the five-frequency experiment in which there were 60 repetitions of each stimulus.

A cued intermixed block was similar to the uncued intermixed block except that each trial contained an auditory cue preceding the presentation of the stimulus.

Each of the three blocks was run per session, with the order of blocks varied from session to session to counteract order effects. Ordinarily there was one session per day and four sessions per experiments, except for the five-frequency experiment in which there were six sessions.
Before each uncued intermixed block, before each cued intermixed block, and before each subblock in the alone block, there were 10 practice trials of each stimulus that would appear in the following block or subblock.

No feedback was provided. All viewing was binocular with natural pupils.

Detalls of each trial. The observers started each two-interval forced-choice trial by pressing a button.

The two temporal intervals in which the stimulus might appear were separated by $190 \mathrm{msec}$ and were each $100 \mathrm{msec}$ long; each interval was identified by a tone. The first of the two intervals began 1200 msec after the subject initiated the trial. Cues, when presented, occurred during this time, with the end of the cue always occurring $760 \mathrm{msec}$ before the beginning of the first interval.

Detalls of the auditory cues. In most experiments, three auditory cues were used, each associated with one of the three possible stimuli. One tone burst indicated the lowest frequency, the left position, or the lowest contrast. Two tone bursts indicated the middle stimulus, and three tone bursts indicated the remaining stimulus. Each tone burst was $68 \mathrm{msec}$ long, and the separation between them was 78 msec.

In the five frequency experiment, the three cues previously described were associated with the three lower frequencies. The nextto-the-highest frequency was indicated by the two shorter tone bursts ( $28 \mathrm{msec})$ separated by a longer duration $(153 \mathrm{msec})$. The highest frequency was indicated by three 28 -msec tone bursts, separated by $153 \mathrm{msec}$.

All observers found it quite easy to discriminate the cues as well as associate each cue with the appropriate stimulus.

\section{Observers}

Three observers, two of whom (E.D. and P.K.) were authors, participated in these experiments. Two observers had normal acuity after correction for myopia. The third, P.K., had a residual deficit accompanied by nystagmus.

\section{RESULTS}

Briefly, addressing in reverse order the four issues in the introduction, the following results were found.

Detectability in cued intermixed blocks was indistinguishable from that in alone blocks. That is, auditory cues preceding the stimulus intervals by about $760 \mathrm{msec}$ eliminated the uncertainty effects where they existed, and there was no evidence of differential pattern adaptation.

Uncertainty effects were not found for contrast but were found for spatial frequency and position.

The uncertainty effects for spatial frequency and position were too small to be explained by singleband models but were consistent with multiple-band models.

\section{Performance in Alone, Uncued Intermixed, and Cued Intermixed Blocks}

The experimental results are shown in Figures 1, 2, and 3 for spatial frequency, position, and contrast, respectively.

In the spatial frequency and position experiments, performance in the uncued intermixed blocks was always worse than in the alone blocks. The difference in percent correct between uncued intermixed and 


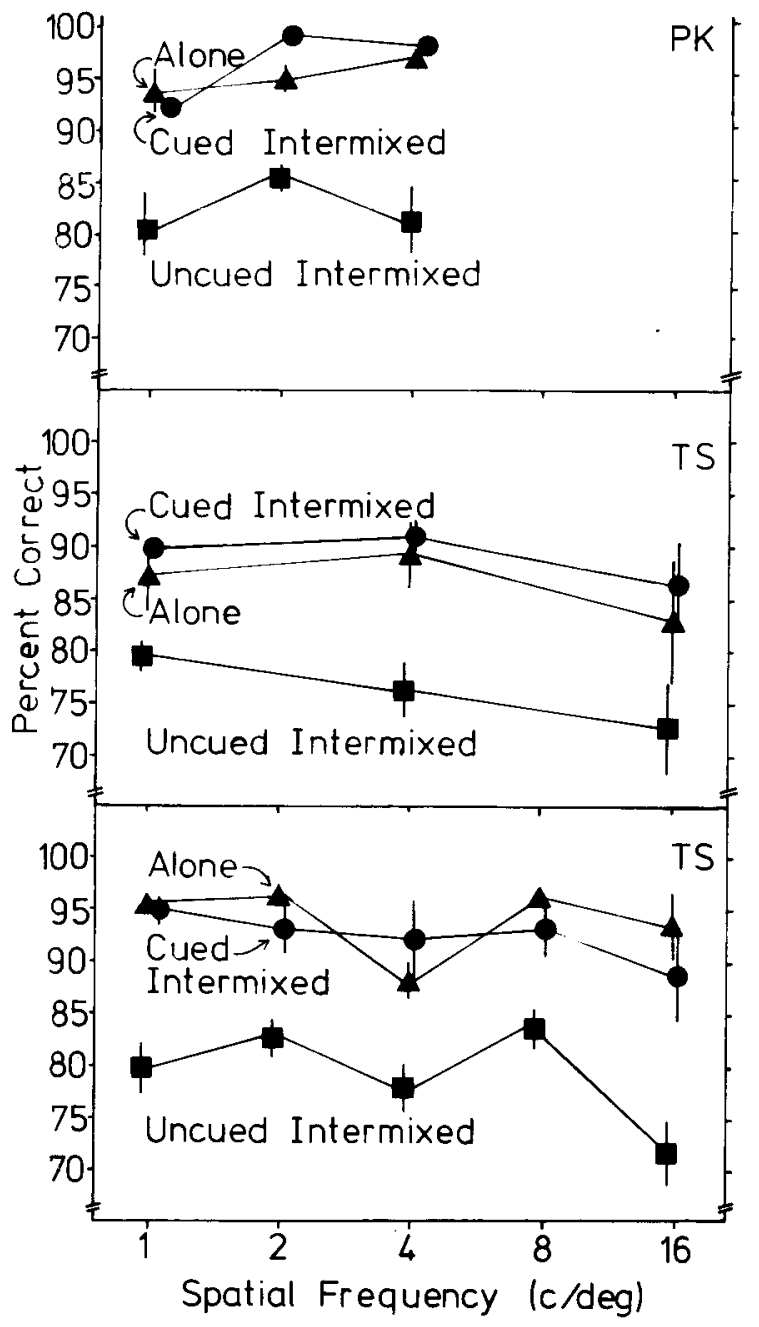

Figure 1. Results from the apatial-frequency uncertalnty experfmentw. Percent correct is plotted on the vertical axis. Spatial frequency is plotted on the horizontal adw. Each panel shows the results from one experiment for the alone condition (triangles), the ancued intermixed condition (squares), and the cued intermbed condition (circles). Each point gives the mean value of percent correct averaged acroas seations, and the vertical bass show plus and minus one standard error of the mean. The observer's initinls are shown in the upper ifght corner of each panel.

alone blocks averaged $13.02 \%$, with a standard error of $.97 \%$ (averaged across all spatial frequencies and positions for all observers). Performance in the cued intermixed blocks was effectively identical to that in the alone blocks. The difference between cued intermixed and alone blocks averaged $.35 \%$, with a standard error of $.72 \%$. Cues preceding the first temporal interval by $760 \mathrm{msec}$ therefore eliminated the uncertainty effect where it existed. Performance in the cued intermixed block was never better, however, than performance in the alone block as would have been expected from differential pattern adaptation. One study of spatial position uncertainty has reported better performance in cued than in alone con- ditions, but they used suprathreshold stimuli which might have produced differential adaptation (Posner, Snyder, \& Davidson, 1980).

In the contrast experiments (Figure 3), performance was essentially identical in all three types of blocks. The difference in percent correct between the uncued intermixed and alone blocks averaged $1 \%$ with a standard error of $1.29 \%$, and the difference between cued intermixed and alone blocks averaged $.44 \%$ with a standard error of $.61 \%$.

\section{Interval Blases}

Although a forced-choice procedure eliminates certain kinds of response bias, it does allow biases toward reporting one interval more often than another. According to the standard version of signal detection theory (Green \& Swets, 1966, p. 410), such a bias will depress overall percent correct. We recalculated results for the worst interval biases, producing "corrected" percent correct responses. The corrected values were usually one or two percentage points higher than uncorrected values, but showed the same pattern of results as the uncorrected values.

\section{Possible Eye Movement and \\ Accommodation Artifact.}

Foveal sensitivity to 2 and $4 \mathrm{cpd}$ is higher than peripheral sensitivity. Observers in the alone and cued intermixed blocks of the spatial-position experiments therefore might have tended on each trial to fixate

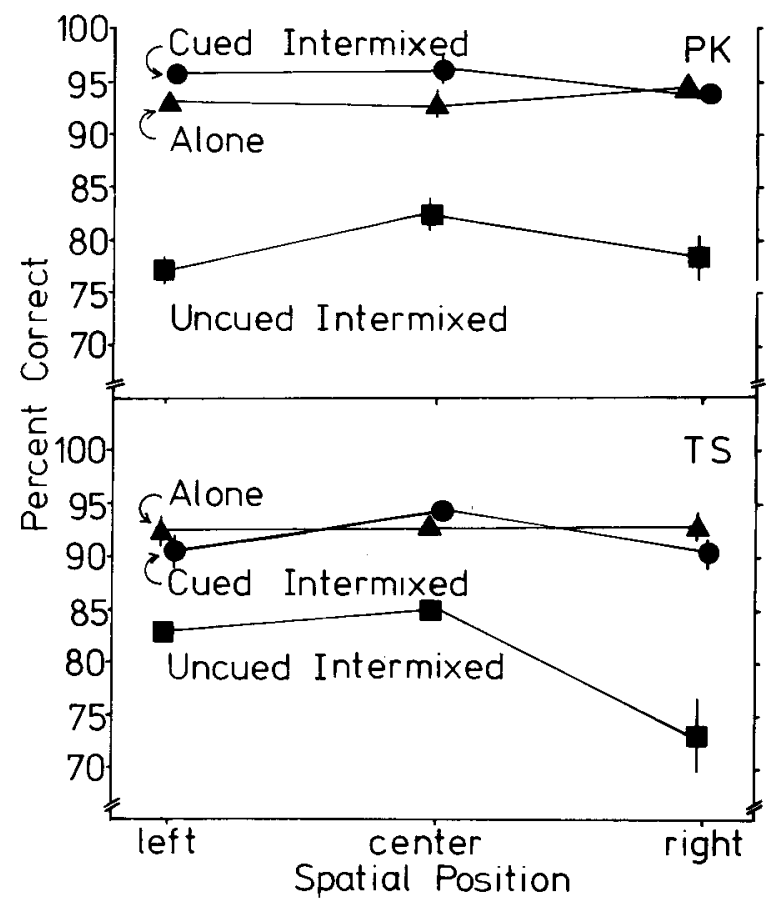

Figure 2. Reaulte from the upatial-poaition uncertainty experlments. The podition of the grating bs shown on the horizontal axis. All other conventions are the anme as in Figare 1. 


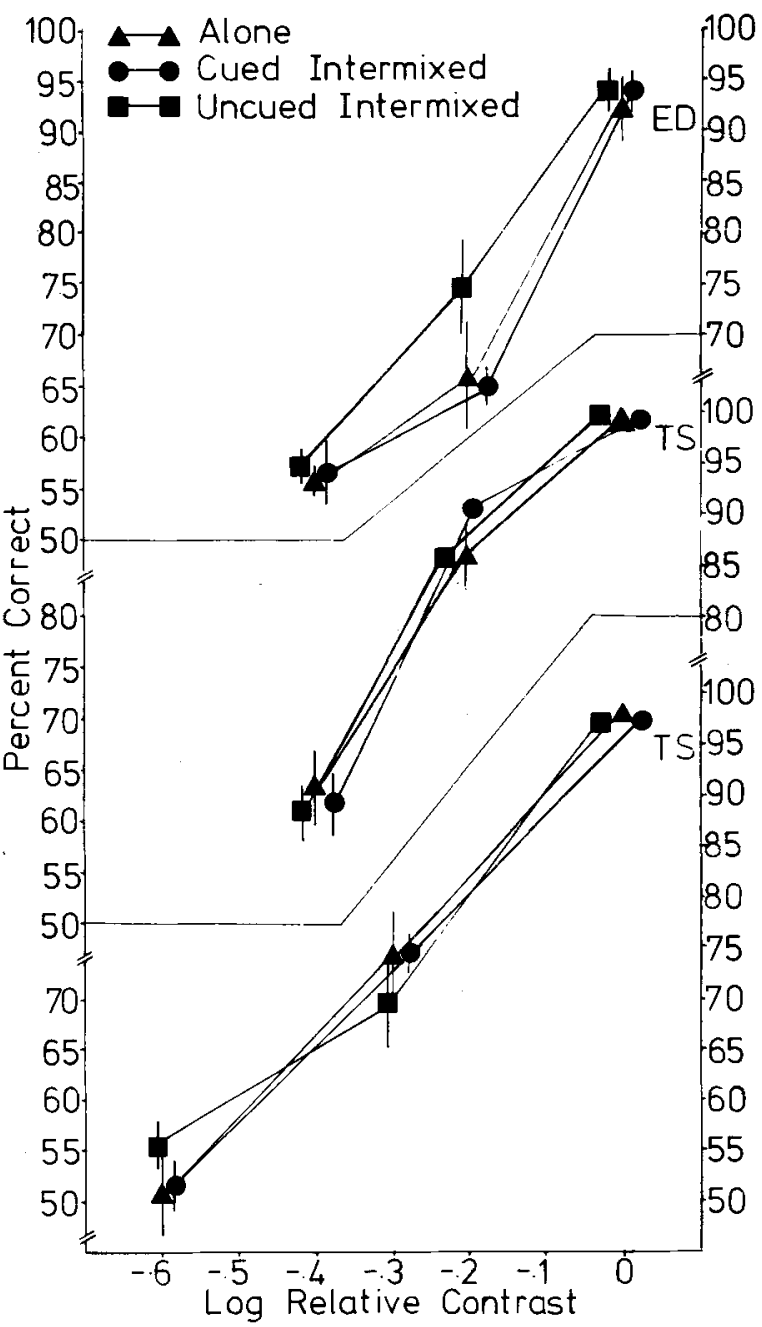

Figure 3. Results from the contrast uncertainty experiments. Relative contrast is given on the horizontal axis. All other conventions are the same as in Figures 1 and 2.

where they knew the stimulus would appear. As a result of these eye movements, the observers could then do better in the alone and cued intermixed blocks than in the uncued intermixed blocks. Our observers, however, were practiced observers who were trying very hard to fixate and who believed they did fixate on almost every trial. Available evidence suggests that observers can fixate very well (e.g., Murphy, Kowler, \& Steinman, 1975) and, although expectations can cause eye movements (Kowler \& Steinman, 1979a, 1979b), the size of these eye movements is too small to have produced our effects. Therefore, although eye movements cannot be ruled out as a potential artifact in the spatial-position experiments, they probably are not responsible for the greatest part of the uncertainty effects. In any case, eye movements cannot account for the very similar effects found in the spatial-frequency uncertainty experiments.
Changes in accommodation have frequently been suggested to us as an explanation for the spatialfrequency uncertainty effects. They seem very unlikely, however, for reasons discussed in Davis and Graham (1981), to be the real explanation.

\section{Session-to-Session Variability}

The variability from session to session is somewhat larger than would be expected if the results from all sessions were random, independent samples from the same populations. Thus, a number of error bars in Figures 1-3 are slightly larger than predicted from the binomial distribution with 400 trials per point (or 360 trials per point in the case of the five-frequency experiment). However, this session-to-session variability did not appear to be systematic; there were no trends over time in performance in any of the conditions.

\section{Comparison with Model Predictions}

Single-band models. A switching single-band model assumes that the observer monitors only one mechanism on a given trial and, if more than one stimulus is presented, the observer may switch his attention from one mechanism to another on different trials. This switching could be the result of limited capacity; that is, the observer may be unable to monitor more than one mechanism at a time.

Performance in an uncued intermixed block is predicted from the following equation:

$$
P_{i}^{*}(C)=a P_{i}(C)+(1-a)(.5),
$$

where $\mathrm{P}_{\mathrm{I}}^{\prime}(\mathrm{C})$ is the probability of correctly reporting a given stimulus, $i$, under conditions of uncertainty; $\mathrm{P}_{\mathrm{i}}(\mathrm{C})$ is the probability of correctly reporting a given stimulus, $i$, under conditions of certainty; and $a$ is the proportion of trials on which the observer monitors the mechanism sensitive to that stimulus. Notice that for the proportion of trials on which the observer does not monitor the relevant mechanism $(1-a)$, he is correct only by chance. Performance in detecting one stimulus can be improved by increasing the proportion of trials on which the observer monitors the relevant mechanism (that is, increasing the value of a). This improvement in detecting one stimulus in an uncued intermixed block, however, will make performance in detecting other stimuli much worse. In any case, the switching single-band model always predicts uncertainty effects much larger than those found for spatial frequency or position. (For example, in the three-spatial-positions experiment of Observer P.K., the probabilities predicted for the three positions in the uncued intermixed blocks are $.65, .64$, and .65 -assuming the observer monitors each mechanism on one-third of the trials. The actual probabilities are .77, .82, and .79.) 
Multiple-band models. According to multipleband models, the observer can simultaneously monitor many mechanisms but does not have to. On every trial in an uncued intermixed block, the observer monitors all the mechanisms that are sensitive to any of the stimuli. On every trial in an alone or cued intermixed block, however, the observer monitors only the one relevant mechanism. Furthermore, each mechanism responds the same way to a given stimulus no matter how many other mechanisms are being monitored.

We considered two quantitative versions of a multiple-band model, both making the conventional assumption that a mechanism's response to a stimulus is normally distributed with the same variance as for the blank. We also assume that there is independent variability in the response of different mechanisms so that each mechanism sometimes produces false alarms to the blank stimulus. Thus, when more mechanisms are monitored there are more opportunities for false alarms. Exactly how multiple-band models predict an uncertainty effect depends on further assumptions of the model.

Adding-of-outputs version. As the name implies, the outputs within each interval are added, and then the observer chooses the interval producing the larger sum. The $d^{\prime}$ value in the uncued intermixed block will equal the $d^{\prime}$ value in the alone block divided by the square root of $n$, where $n$ is the number of independent mechanisms monitored in the uncued intermixed block. In our experiments, $n$ was taken to equal the number of stimuli used. [The derivation of this prediction is given in several sources-such as Creelman (1960) and Ball and Sekuler (1981). We used the conversion between $d^{\prime}$ and two-interval forced choice percent correct from Elliot's Table II in Appendix I of Swets (1964).]

Maximum-output version. This version of a multiple-band model assumes that the observer's response is based on the maximum of the outputs from all the monitored channels. In a forced-choice experiment, the observer chooses the interval in which the maximum output is larger. [Our calculations for this model used Elliot's table in Appendix I of Swets (1964) to give the probability that the largest of $M$ outputs was from the correct channel. Alternative calculations are described in Green and Weber (1980). This model is a very good approximation to an ideal-observer model-the model in which the observer uses information from the multiple mechanisms in the optimal way (Nolte \& Jaarsma, 1967; and see further discussion in Green \& Weber, 1980).]

Other multiple-band models are possible, of course. The shape of the distributions need not be Gaussian and rules other than adding-of-outputs and maximum-output can be formulated. Several of these alternatives are discussed in Green and Weber (1980) and Lappin and Staller (1981). However, these two are sufficient for our present purposes.
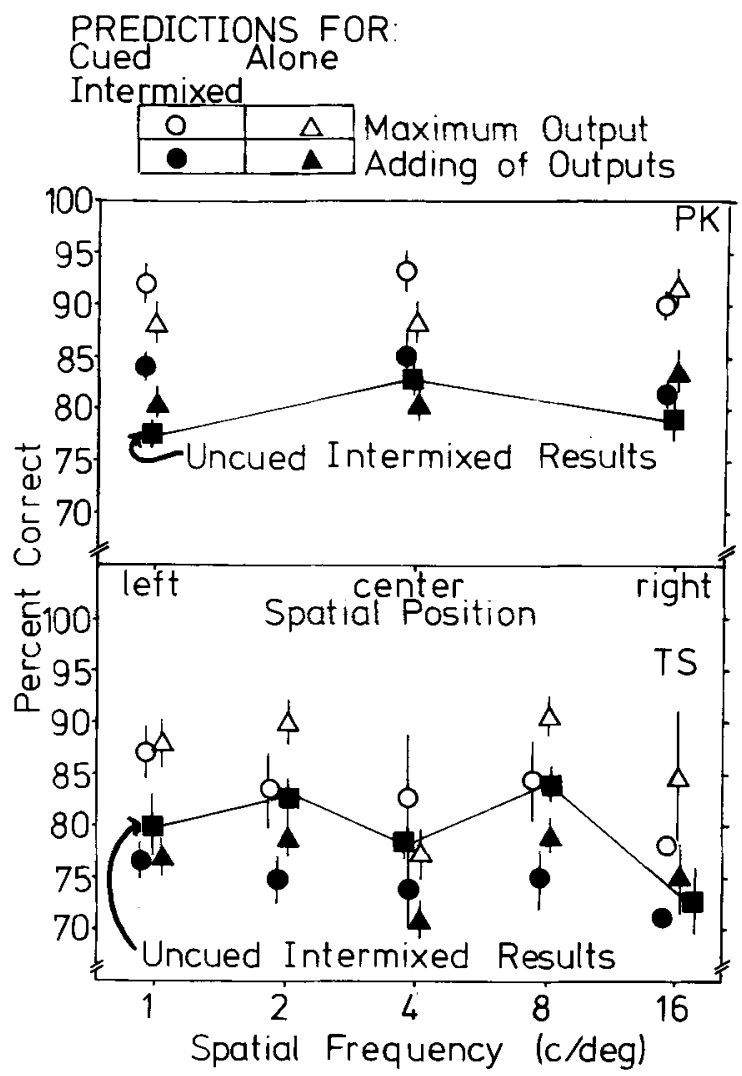

Figure 4. Comparison of experimental results with theoretical predictions. The results for the uncued intermixed condition from two experiments (the spatial-podtion experiment whth Obeerver P.X. and the spatial-frequency experiment with Obaerver T.S. and five frequenciea) are replotted from Figures 1 and 2 as illed squares connected by a solid line. The other aymbols show the predictions for the uncued Intermbed condition from two verions of the moltiple-band model (the moximam ontpat venion as open arm. bols, the adding-of-output venton as closed eymboln) calculated from elther the results in the cued intermbred condition (circlen) or the reault in the alone condidion (triangles). The error barn on the predictions are explained in the text.

All our spatial-frequency and spatial-position results were consistent with at least one of these two versions of a multiple-band model. Figure 4 shows the comparison of model predictions with the results from the spatial-position experiment for $\mathrm{Ob}$ server P.K. (top panel) and the five spatial-frequencies experiment for Observer T.S. (bottom panel). Maximum-output (open symbols) and adding-of-outputs (closed symbols) predictions for the uncued-intermixed blocks were computed from mean percent correct in the alone blocks (triangles) and in the cued-intermixed blocks (circles). (Since errors of measurement in the alone and cued-intermixed results propagate through the calculations and cause imprecision in the predictions, predictions were also computed from the means plus-or-minus one standard error and are represented by vertical bars through the symbols.) Notice that the maximum-output model predicts a 
substantially smaller uncertainty effect than the addingof-outputs model.

Of the five experiments producing uncertainty effects, three (the two spatial-frequency experiments and the one spatial-position experiment for $\mathrm{Ob}$ server T.S.) produced results like those in the bottom panel of Figure 4. For these three experiments, the uncertainty effects tended to be somewhat smaller than predicted by the adding-of-outputs version and somewhat larger than predicted by the maximumoutput version, but neither version could be confidently rejected.

The other two experiments (the spatial-frequency and spatial-position experiments for Observer P.K.) showed results like those in the top panel of Figure 4. The uncertainty effects were always as large as those predicted by the adding-of-outputs version and larger than those predicted by the maximum-output version.

These two different patterns of results may represent a true difference between these two observers. Since the differences are small, however, in addition to the fact that the results for the two observers were not consistent, we do not feel safe in concluding in favor of either version of the multiple-band model for either observer. We do feel safe in concluding that the magnitude of the effects is consistent with multiple-band, but not single-band, models.

Intermediate models. The size of the uncertainty effects would also be consistent with intermediate models in which most, but not all, of the relevant mechanisms are monitored on every trial and the observer's response is based on the maximum output of the monitored mechanisms. In principle, examining sequential conditional probabilities might distinguish this intermediate model (where switches from trial to trial in which mechanisms were monitored might introduce sequential dependencies) from a pure multiple-band model. In fact, the sequential dependencies found in this study were very weak, consistent with a rejection of the switching single-band model. ${ }^{1}$ What sequential dependency there was might result from factors such as slow temporal shifts in the sensitivity of all mechanisms and does not seem good evidence for an intermediate over a pure multipleband model.

\section{DISCUSSION}

\section{Implications for Models of Pattern Vision}

Uncertainty effects were found for spatial frequency and spatial positions (Figures 1 and 2) but not for contrast (Figure 3). The practical implications for psychophysical experiments measuring the detectability of simple patterns is clear. Whether or not contrasts are intermixed probably does not matter; whether or not spatial frequencies and positions are intermixed does.

Some theoretical implications are also clear. Cur- rent theories of pattern vision assume mechanisms selectively sensitive to spatial frequency and to position but not to contrast. The present results lend support to this assumption. (Finding no contrast uncertainty effect, of course, is only evidence against mechanisms sensitive to ranges of contrast narrower than that used here- .6 log units. Mechanisms selectively sensitive to broader ranges of contrast cannot be ruled out.)

Furthermore, in current theories of pattern vision, the mechanisms selectively sensitive to spatial frequency (perhaps arrays of receptive fields identical in size and orientation, but differing in position) and those selectively sensitive to position (perhaps sets of receptive fields differing in size and orientation but all at the same position) are assumed to have very similar properties. In fact, it is frequently simpler to talk of one set of mechanisms (single receptive fields), each of which is sensitive to a limited range of spatial frequencies and to a limited range of positions (as, for example, is done by Wilson \& Bergen, 1979). On the basis of these models, one would expect the effects of uncertainty on these two dimensions to be quite similar-as they turned out to be-as long as the grating patches are far enough apart in spatial frequency (a factor of approximately 2) or in spatial position (centered approximately 2 or 3 cycles apart) to effectively stimulate different receptive fields.

Probabilistically independent mechanisms for spatial frequency and spatial position. The results of summation experiments (e.g., Graham, Robson, \& Nachmias, 1978; Robson \& Graham, 1981; Watson 1982), in which the detectability of compound patterns is compared with that of the components alone, are consistent with probability summation due to independent variability in the responses of different mechanisms. However, an alternative interpretation in terms of nonlinear pooling is possible (see below). Certain results from spatial recognition experiments (Hirsch, Hylton, \& Graham, 1982) also suggest some independent variability, but these results are quite complicated. Independent variability is at the heart of the ability of the above multiple-band models to account for uncertainty effects. That the size of the spatial-frequency and spatial-position uncertainty effects is consistent with multiple-band models (Figure 4) is, therefore, further support for the existence of independent variability in the responses of different mechanisms.

Quick pooling model. At this point, however, the paradox of the Quick pooling model arises. It is this particular model of independent variability in the responses of different mechanisms that has been so successful in accounting for the thresholds of many kinds of patterns, including those from the summation experiments mentioned above. Yet, since it is a high-threshold model in which there is no pos- 
sibility of an individual channel's producing a false alarm, it predicts that there should be no effect of uncertainty. Furthermore, high-threshold models make incorrect predictions about the psychometric functions for sinusoidal gratings (Nachmias, 1981) as well as for other stimuli (Green \& Swets, 1966). In detail, therefore, the Quick pooling model has to be wrong.

Why, then, has the Quick pooling model been so extraordinarily successful in accounting for the thresholds of a wide variety of patterns? One possible answer is that the correct derivation of the principle formula of the Quick pooling model is not from a high-threshold model at all. An alternative derivation has been suggested (Quick, 1974). It assumes that deterministic signals from multiple mechanisms are pooled in a nonlinear fashion to form one signal. To account for the variability present in the psychophysical response, a second stage must be assumed at which variability is added to this pooled signal.

Under this alternative derivation of the Quick pooling formula, however, there is still no entirely satisfactory way to explain uncertainty effects. Since only one source of variability exists (added at the second stage), multiple-band explanations are not applicable. If some kind of capacity limitation is suggested instead, the agreement found between our uncertainty results and predictions of multiple-band models would have to be mere coincidence. Furthermore, the agreement found in other studies between the slope of the psychometric function and the amount of summation would have to be another coincidence. (See Robson \& Graham, 1981, for further discussion of this point.) Thus, invoking the alternative derivation of the Quick pooling formula does not seem a good answer to the question of why the Quick pooling model has been so successful.

An approach to a more satisfying answer may come from comparing the successes of the model to its failures. The Quick pooling model has succeeded when pooling across several mechanisms was involved (as in relating the thresholds of different patterns to one another in summation experiments); it has failed when a single mechanism was predominantly important (as in comparing the detectability of the same pattern in alone vs. uncued-intermixed blocks). The successful predictions may depend primarily on the assumptions relating different mechnisms to one another (independent variability and a maximum output rule-assumptions shared with the maximum output version of the multiple-band model above). They may not depend very much on the assumptions about each mechanism's response (that there is a high threshold).

Uncertainty about form. One previous study failed to find an effect of uncertainty about spatial form (Posner, Snyder, \& Davidson, 1980), a variable that might be expected to act like spatial frequency. The forms in question, however, were different letters of the alphabet. All letters, in fact, contain many of the same spatial frequencies.

\section{Comparison with Uncertainty \\ in Other Dimensions}

In general, the effects of uncertainty about attributes of stimuli may well differ, depending on the manner in which those stimuli are being handled by the nervous system (e.g., Egeth, 1977; Erdelyi, 1974; Lappin \& Staller, 1981; Lappin \& Uttal, 1976; Shaw, 1980). This, in turn, will depend both on the characteristics of the stimuli and on the observer's task, both as it is explicitly defined and as it is influenced by other aspects of the experimental procedure.

Two situations in which uncertainty effects have been studied seem sufficiently like the situations here to be worth commenting on. Theories about auditory frequency and about the direction of motion of simple visual patterns (reviewed in Oreen, 1976, and Sekuler, Pantle, \& Levinson, 1978, respectively) are similar to the current theories about spatial frequency and spatial position. In particular, they postulate selectively sensitive mechanisms.

Investigations of auditory-frequency uncertainty effects have been somewhat inconclusive. There is a brief review in Ball and Sekuler (1980) as well as Swets and Kristofferson (1970). In a similar study, however, Creelman (1960) reached conclusions very similar to ours.

The effect of uncertainty about the direction of motion of simple low-contrast patterns seems to be much larger than is predictable from multiple-band models (Ball \& Sekuler, 1980) although consistent with single-band models. When two different directions were intermixed in a block, in fact, observers seemed to monitor a single mechanism sensitive to the direction midway between the two presented.

This difference between the effects of uncertainty about the direction of motion of low-contrast moving patterns and the effects of uncertainty about spatial frequency and spatial position of gratings is surprising, since, in so many ways, effects on these two dimensions have been similar. Perhaps the implicit task demands in the two situntions were different. In some situntions, observers do act as if they were monitoring only a few spatial-frequency channels (Davis \& Graham, 1981). If, however, observers could have monitored more than a single direction-of-motion mechanism, for optimal performance they certainly should have, particularly in the forced-choice detection experiment most similar to the ones here (Experiment 6 of Ball \& Sekuler, 1980²).

\section{RMFERENCES}

Ball, K., \& Bexulen, R. Models of stimulus uncertainty in motion perception. Psychological Review, 1980, 07, 435-469. BAlL, K., \& Sexulen, R. Cues reduce direction uncertainty 
and enhance motion detection. Perception \& Psychophysics, 1981, 30, 119-128.

Bergen, J. R., Wilson, H. R., \& Cowan, J. D. Further evidence for four mechanisms mediating vision at threshold: Sensitivities to complex gratings and aperiodic stimuli. Journal of the Optical Society of America, 1979, 69, 1580-1586.

Blakemore, C., \& Campeell, F. W. On the existence of neurones in the human visual system selectively sensitive to the orientation and size of retinal images. Journal of Physiology (London), 1969, 203, 237-260.

CoHn, T. E., \& LASLEY, D. J. Detectability of a luminance increment: Effect of spatial uncertainty. Journal of the Optical Society of America, 1974, 64, 1715-1719.

Creelman, C. D. Detection of signals of uncertain frequency. Journal of the Acoustical Society of America, 1960, 32, 805-810.

DAvis, E. T. Allocation of attention: Uncertainty effects when monitoring one or two visual gratings of noncontiguous spatial frequencies. Perception \& Psychophysics, 1981, 29, 618-622.

Davis, E. T., \& Graham, N. Spatial frequency uncertainty effects in the detection of sinusoidal gratings. Vision Research, 1981, 21, 705-712.

EGeTH, H. Attention and preattention. In G. H. Bower (Ed.), The psychology of learning and motivation (Vol. 11). New York: Academic Press, 1977.

Erderly, M. H. A new look at the new look: Perceptual defense and vigilance. Psychological Review, 1974, 81, 1-25.

Gilliom, J. D., \& Milss, W. M. Information extraction from contralateral cues in the detection of signals of uncertain frequency. Journal of the Acoustical Society of America, 1976, 59, 1428-1433.

GrahaM, N. Visual detection of aperiodic spatial stimuli by probability summation among narrowband channels. Vision Research, 1977, 17, 637-652.

Graham, N. The visual system does a crude Fourier analysis of patterns. In S. Grossberg (Ed.), Mathematical psychology and psychophysiology (SIAM-AMS Proceedings; Vol. 13). Providence, R.I: American Mathematical Society, 1981.

Graham, N., \& Nachmias, J. Detection of grating patterns containing two spatial frequencies: A comparison of singlechannel and multiple-channels models. Vision Research, 1971, 11, 251-259.

Graham, N., Robson, J. G., \& Nachmias, J. Grating summation in fovea and periphery. Vision Research, 1978, 18, 815-825.

Green, D. M. An introduction to hearing. Hillsdale, N.J: Erlbaum, 1976.

GreEN, D. M., \& LUCE, R. D. Variability of magnitude estimates: A timing theory analysis. Perception \& Psychophysics, 1974, 15, 291-300.

Green, D. M., \& Swets, J. A. Signal detection theory and psychophysics. New York: Wiley, 1966.

Green, D. M., \& Weben, D. L. Detection of temporally uncertain signals. Journal of the Acoustical Society of America, 1980, 67, 1304-1311.

Hirsch, J., Hylton, R., \& Graham, N. Simultaneous recognition of two spatial-frequency components. Vision Research, 1982, 22, 365-375.

Kowlen, E., \& Steinman, R. M. The effect of expectations on slow oculomotor control. I. Periodic target steps. Vision Research, 1979, 19, 619-632. (a)

Kowler, E., \& Steinman, R. M. The effect of expectations on slow oculomotor control. II. Single target displacements. Vision Research, 1979, 19, 633-647. (b)

Lappin, J. S., \& Staller, J. D. Prior knowledge does not facilitate the perceptual organization of dynamic random-dot patterns. Perception \& Psychophysics, 1981, 29, 445-456.

LAPPIN, J. S., \& UTTAL, W. R. Does prior knowledge facilitate the detection of visual targets in random noise? Perception \& Psychophysics, 1976, 20, 367-374.

Luce, R. D., Green, D. M., \& Weber, D. L. Attention bands in absolute identification. Perception \& Psychophysics, 1976, 20, 49-54.
Mostafavi, H., \& Sakrison, D. J. Structure and properties of a single channel in the human visual system. Vision Research, 1976, 16, 957-968.

Murphy, B. J., Kowler, E., \& Steinman, R. M. Slow oculomotor control in the presence of moving backgrounds. Vision Research, 1975, 15, 1263-1268.

Nachmiss, J. On the psychometric function for contrast detection. Vision Research, 1981, 21, 215-223.

Nolte, L. W., \& JaArsma, D. More on the detection of one of $\mathrm{M}$ orthogonal signals. Journal of the Acoustical Society of America, 1967, 41, 497-505.

Pantle, A., \& Sekuler, R. Size-detecting mechanisms in human vision. Science, 1968, 162, 1146-1148.

Pelli, D. G. The effect of uncertainty: Detecting a signal at one of ten-thousand times and places. In Supplement to Investigative Ophthalmology and Visual Science, 1981, 20, 3, 178.

Posner, M. I. Chronometric explorations of mind. Hillsdale, N.J: Erlbaum, 1978.

Posner, M. I., Snyder, C. R. R., \& Davidson, B. J. Attention and the detection of signals. Journal of Experimental Psychology: General, 1980, 109, 160-174.

Quick, R. F. A vector-magnitude model for contrast detection. Kybernetik, 1974, 16, 65-67.

Quick, R. F., Mullins, W. W., \& Reichert, T. A. Spatial summation effects on two-component grating thresholds. Journal of the Optical Society of America, 1978, 68, 116-121.

Robson, J. G., \& Graham, N. Probability summation and regional variation in contrast sensitivity across the visual field. Vision Research, 1981, 21, 409-418.

Sexuler, R., Pantle, A., \& Levinson, E. Physiological basis of motion perception. In R. Held, H. W. Leibowitz, \& H. L. Teuber (Eds.), Handbook of sensory physiology (Vol. VIII): Perception. New York: Springer, 1978.

Shaw, M. Identifying attentional and decision-making components in information processing. In R. S. Nickerson (Ed.), Attention and performance VIII. Hillsdale, N.J: Erlbaum, 1980.

SwETs, J. A. Signal detection and recognition by human observers. New York: Wiley, 1964.

Swets, J. A., \& Kristorferson, A. B. Attention. Annual Review of Psychology, 1970, 21, 339-366.

Swets, J. A., \& Sewalt, S. T. Stimulus vs response uncertainty in recognition. Journal of the Acoustical Society of America, 1961, 33, 1586-1592.

Watson, A. B. Summation of grating patches indicates many types of detector at one retinal location. Vision Research, $1982,22,17-26$.

Wilson, H. R., \& Bergen, J. R. A four mechanism model for threshold spatial vision. Vision Research, 1979, 19, 19-32.

\section{NOTES}

1. Sequential dependencies found in the uniform intermixed blocks of an earlier study (Davis \& Graham, 1981) were stronger and statistically significant. The overall conditions of that study, including the unbalanced intermixed blocks and the practice trials of the primary stimulus before each block, may have encouraged the observers to follow an intermediate model (i.e., to monitor small groups of channels) even in the uniform intermixed blocks.

2. Performance levels in the two studies were different $[75 \%$ correct for Experiment 6 , of Ball and Sekuler (1980) and greater than $90 \%$ correct here in the alone blocks]. However, the midway model continues to describe suprathreshold reaction times to different directions of motion (Ball \& Sekuler, 1980) and results from subsequent spatial frequency uncertainty experiments done in our laboratory at lower performance levels were consistent with multiple-band, not single-band, models.

(Manuscript received April 21, 1982; revision accepted for publication September 23, 1982.) 